\title{
Precision calculations in the MSSM Higgs-boson sector with FeynHiggs 2.14
}

\author{
H. Bahl ${ }^{\mathrm{a}, \mathrm{b}, *}$, T. Hahn ${ }^{\mathrm{b}}$, S. Heinemeyer ${ }^{\mathrm{c}}$, W. Hollik ${ }^{\mathrm{b}}$, \\ S. Paßehr ${ }^{\text {d }}$ H. Rzehak ${ }^{\mathrm{e}}$, G. Weiglein ${ }^{\mathrm{a}}$ \\ ${ }^{a}$ DESY, Notkestraße 85, D-22607 Hamburg, Germany \\ ${ }^{b}$ Max-Planck-Institut für Physik, Föhringer Ring 6, D-80805 Munich, Germany \\ ${ }^{c}$ Instituto de Fúsica Teórica, Universidad Autónoma de Madrid Cantoblanco, 28049 Madrid, Spain; \\ Campus of International Excellence UAM+CSIC, Cantoblanco, 28049, Madrid, Spain; \\ Instituto de Física de Cantabria (CSIC-UC), E-39005 Santander, Spain \\ ${ }^{d}$ Sorbonne Université, CNRS, Laboratoire de Physique Théorique et Hautes Énergies (LPTHE), \\ 4 Place Jussieu, F-75252 Paris CEDEX 05, France \\ ${ }^{e}$ CP3-Origins, University of Southern Denmark, Odense, Denmark
}

\begin{abstract}
We present an overview of the status and recent developments of FeynHiggs (current version: 2.14.3) since version 2.12.2. The main purpose of FeynHiggs is the calculation of the Higgs-boson masses and other physical observables in the MSSM. For a precise prediction of the Higgs-boson masses for low and high SUSY scales, state-of-the-art fixed-order and effective-field-theory calculations are combined. We first discuss improvements of the fixed-order calculation, namely an optional $\overline{\mathrm{DR}}$ renormalization of the stop sector and a renormalization of the Higgs sector ensuring the chosen input mass to be equivalent with the corresponding physical mass. Second, we describe improvements of the EFT calculation, i.e. an implementation of non-degenerate threshold corrections as well as an interpolation for complex parameters. Lastly, we highlight some improvements of the code structure easing future extensions of FeynHiggs to models beyond the MSSM.
\end{abstract}

Keywords: MSSM, Higgs-boson observables;

\footnotetext{
* Corresponding author.

E-mail address: henning.bahl@desy.de
} 


\section{New version program summary}

Program Title: FeynHiggs

Licensing provisions: GPLv3

Programming language: Fortran, C, Mathematica

Journal reference of previous version: Comput. Phys. Comm. 180 (2009) 1426

Does the new version supersede the previous version? Yes.

Reasons for the new version: Improved calculations and code structure.

Summary of revisions: Apart from improvements discussed in other publications: implementation of optional $\overline{\mathrm{DR}}$ renormalization of stop sector, adapted two-loop Higgs sector renormalization, implementation of full non-degenerate threshold corrections, interpolation of EFT calculation for complex parameters, better code structure.

Nature of problem: The Minimal Supersymmetric Standard Model (MSSM) allows predictions for the masses and mixings of the Higgs bosons in terms of a few relevant parameters. Therefore, comparisons to experimental data provide constraints on the parameter space. To fully profit from the experimental precision, a comparable level of precision is needed for the theoretical prediction.

Solution method: State-of-the-art fixed-order and effective-field-theory calculations are combined to obtain a precise prediction for small as well as large supersymmetry scales. 


\section{Contents}

1 Introduction

2 FeynHiggs overview

2.1 Higgs boson mass spectrum . . . . . . . . . . . . . . . 5

2.2 Other observables . . . . . . . . . . . . . . . . . . 6

2.3 Using FeynHiggs . . . . . . . . . . . . . . . . . . . 7

3 Improvements of the fixed-order calculation 9

3.1 Optional $\overline{\mathrm{DR}}$ renormalization . . . . . . . . . . . . . . . . . 9

3.2 Adapted renormalization of the Higgs sector . . . . . . . . . . . . . . 11

4 Improvements of the EFT calculation 13

5 Improvements of code structure $\quad 14$

6 Numerical results 17

6.1 Improvements of the fixed-order calculation . . . . . . . . . . . 17

6.2 Improvements of the EFT calculation . . . . . . . . . . . . . . 18

7 Conclusions $\quad 20$ 


\section{Introduction}

While the gauge sector of the Standard Model (SM) is well investigated, the experimental precision in the Higgs sector [1-4] leaves significant room for physics beyond the SM (BSM). One of the most frequently discussed BSM theories containing an extended Higgs sector is the Minimal Supersymmetric Standard Model (MSSM) [5, 6] based upon the concept of supersymmetry (SUSY). Apart from adding a superpartner to every SM degree of freedom, the MSSM also introduces a second Higgs doublet resulting in five physical Higgs bosons: at the tree level, these are the $\mathcal{C P}$-even $h$ and $H$ bosons, the $\mathcal{C} \mathcal{P}$-odd $A$ boson as well as the charged $H^{ \pm}$bosons. Owing to the underlying supersymmetry, the Higgs sector is determined by only two additional nonSM parameters at the tree level. Conventionally, they are chosen as the ratio of the vacuum expaction values (vevs) of the two doublets, $\tan \beta=v_{2} / v_{1}$, and the mass of the $A$-boson, $M_{A}$ (or the mass of the charged bosons, $M_{H^{ \pm}}$).

Consequently, the Higgs sector of the MSSM is highly predictive, i.e. the increasingly precise measurements of the properties of the Higgs boson discovered by the ATLAS and CMS collaborations [1,2] at the Large Hadron Collider (LHC) allows one to efficiently probe the parameter space of the MSSM by comparing the high-precision measurements of Higgs-boson properties to the corresponding theoretical predictions.

In order to perform a meaningful comparison, it is essential to take into account radiative corrections to the theory predictions, since these have a large impact on the Higgs sector of the MSSM. To obtain a prediction with an uncertainty comparable to the experimental precision, much work has been dedicated to the calculation of these corrections (for the MSSM with real parameters see [7 68], for the MSSM with complex parameters see [43, 49, 69, 83]).

Apart from tackling the actual calculations, there has also been a major effort to make the results publicly available by providing them in terms of easily usable computer programs.

One such program is FeynHiggs [26, 38, 52, 60, 63, 77, 84, 85], which is available at http://feynhiggs.de. Its main purpose is the calculation of the Higgs-boson masses in the MSSM, amended by predictions for various other phenomenologically relevant observables. It has become a standard tool that is used for instance by the LHC Higgs Cross-Section Working Group [86, 87]. For the calculation of the Higgs boson masses a combined approach of fixed-order Feynman-diagrammatic and effective-field-theory (EFT) calculations is employed. In this paper, we describe recent updates of the code released in versions 2.13.0 through 2.14.3. They improve both the diagrammatic calculation (see Section 3) and the EFT calculation (see Section 4). Moreover, we discuss improvements of the code structure (see Section 5) and give a short introduction to FeynHiggs and how to use it (see Section 2). In the recent version FeynHiggs 2.14.0, also the pole-mass-determination procedure was improved. This issue will, however, be discussed in a separate upcoming publication [88]. 


\section{FeynHiggs overview}

\subsection{Higgs boson mass spectrum}

One of the main purposes of FeynHiggs is to provide predictions for the Higgs-boson masses in the MSSM. The most direct approach is to calculate higher-order corrections to the propagators of the Higgs bosons performing a fixed-order Feynman-diagrammatic calculation. FeynHiggs was originally developed around this approach: It incorporates full one-loop contributions [15, 18, 21] as well as the leading two-loop contributions 1 ] of $\mathcal{O}\left(\alpha_{t} \alpha_{s}, \alpha_{b} \alpha_{s}, \alpha_{t}^{2}, \alpha_{t} \alpha_{b}, \alpha_{b}^{2}\right)$ [26, 32, 34, 35, 38, 40, 45, 77, 80, 84, 85, 89, 90] to the Higgs two-point functions. For these corrections, a mixed OS/DR renormalization scheme is employed (see [77] for more details). The diagrammatic calculation allows one to take into account complex parameters fully at the one-loop level [77] and at $\mathcal{O}\left(\alpha_{t} \alpha_{s}, \alpha_{t}^{2}\right)$ [7880, 90] at the two-loop level (the phase dependences of the other two-loop corrections are interpolated). Moreover, non-minimal flavour violation can be considered at the one-loop level [91, 93].

The diagrammatic calculation captures all contributions at a given order. This result contains logarithms involving some SUSY mass divided by the mass of a SM particle. For relatively low SUSY scales, these logarithms are small and the fixed-order calculation is therefore expected to be precise. For a large separation between the SUSY scale and the electroweak scale, however, these logarithms become large. Thus, they can spoil the convergence of the perturbative expansion, rendering the fixed-order calculation inaccurate.

Effective-field-theory (EFT) techniques provide a tool to resum these large logarithmic contributions to all orders [53, 55, 57, 59, 62, 67, 68, 94]. The main idea is to integrate out some or all heavy SUSY particles at a high scale. The effective couplings are then evolved down to the electroweak scale at which the Higgs mass (or masses) are calculated, effectively resumming all large logarithms that emerged from the masses of the heavy SUSY particles. A state-of-the-art EFT calculation is available in FeynHiggs [52, 60, 63]: based upon the results of [55, 57, 62], it includes full resummation of leading and next-to-leading logarithms (NLL) as well as $\mathcal{O}\left(\alpha_{s}, \alpha_{t}\right)$ resummation of nextto-next-to-leading logarithms (NNLL). Moreover, it allows one to take into account light electroweakinos and gluinos by implementing the corresponding low-energy thresholds. This logarithmic accuracy level ensures a high precision for high SUSY scales. However, since no higher-dimensional operators are included in the EFT calculation, terms suppressed by the SUSY scale are missed (see the discussion in [62]). Therefore, the EFT calculation can become inaccurate for low SUSY scales.

In order to ensure a precise prediction for low, intermediary, and high SUSY scales, the fixed-order approach and the EFT approach are combined in FeynHiggs [52,60, 63,

\footnotetext{
${ }^{1}$ The two-loop self-energy corrections are computed in the approximation of vanishing electroweak gauge couplings and vanishing external momentum (see however [54, 58, 83] for studies going beyond this approximation).
} 
67. This is achieved by adding the resummed logarithms obtained in the EFT approach to the self-energies obtained in the fixed-order approach and removing the doublecounted logarithms by subtraction terms.

Finally, the renormalized self-energies, $\hat{\Sigma}$, supplemented by the resummed logarithms are used to obtain the pole masses of the Higgs bosons. For the neutral Higgs bosons this means that one has to find the poles of the propagator matrix, whose inverse is given by

$$
\begin{aligned}
& \hat{\Gamma}_{h H A}\left(p^{2}\right)= \\
& \mathrm{i}\left[p^{2} \mathbf{1}-\left(\begin{array}{ccc}
m_{h}^{2} & 0 & 0 \\
0 & m_{H}^{2} & 0 \\
0 & 0 & m_{A}^{2}
\end{array}\right)+\left(\begin{array}{ccc}
\hat{\Sigma}_{h h}\left(p^{2}\right)+\Delta_{h h}^{\operatorname{logs}} & \hat{\Sigma}_{h H}\left(p^{2}\right)+\Delta_{h H}^{\operatorname{logs}} & \hat{\Sigma}_{h A}\left(p^{2}\right) \\
\hat{\Sigma}_{h H}\left(p^{2}\right)+\Delta_{h H}^{\operatorname{logs}} & \hat{\Sigma}_{H H}\left(p^{2}\right)+\Delta_{H H}^{\operatorname{logs}} & \hat{\Sigma}_{H A}\left(p^{2}\right) \\
\hat{\Sigma}_{h A}\left(p^{2}\right) & \hat{\Sigma}_{H A}\left(p^{2}\right) & \hat{\Sigma}_{A A}\left(p^{2}\right)
\end{array}\right)\right] .
\end{aligned}
$$

The mixing with the neutral Goldstone boson and the $Z$ boson yields subleading twoloop contributions to the mass predictions and is therefore neglected. The $\Delta$-terms contain the resummed logarithms, obtained in the EFT approach, as well as the corresponding subtraction terms $2^{2}$ If all input parameters are real, $\hat{\Sigma}_{h A}$ and $\hat{\Sigma}_{H A}$ vanish, and the $(3 \times 3)$ mixing is reduced to a $(2 \times 2)$ mixing.

The real parts of the complex poles yield the physical Higgs-boson masses. The masses are conventionally labelled as $M_{h_{i}}(i=1,2,3)$ in the case of $(3 \times 3)$ mixing, and as $M_{h}, M_{H}$ and $M_{A}$ in the case of $(2 \times 2)$ mixing.

In order to treat external Higgs bosons on-shell (e.g. in decay rates), the (nonunitary) Z-matrix is caculated. [15, 77, 95 97] (see also [98] and Sect. 5.3 of [99]). It relates the tree-level mass eigenstates to the external physical states. Also an approximated form of the $\mathbf{Z}$-matrix is given in the output, the $\mathbf{U}$-matrix. It is by default defined as the unitary matrix diagonalizing the inverse propagator matrix, Eq. (1), in the approximation of vanishing momentum [95, 100] and is used to obtain effective couplings.

FeynHiggs furthermore provides an estimate of the remaining theoretical uncertainties from unknown higher-order corrections for all Higgs boson masses, for the Z-matrix, and for the U-matrix [38].

\subsection{Other observables}

The calculated Higgs masses and the $\mathbf{Z}$-matrix are used as input for the prediction of various other observables in the MSSM. The implemented decay widths are sum-

\footnotetext{
${ }^{2}$ The resummation of large logarithms is so far restricted to the $h h, h H$ and $H H$ self-energies. In the case of the SM as low-energy EFT, the resummation of logarithms in the $h H$ and $H H$ self-energies is approximated by essentially dividing the correction to the $h$ self-energy by $\tan \beta$ and $\tan ^{2} \beta$, respectively (see [52,67] for more details).
} 


\begin{tabular}{|c|c|c|}
\hline decay width $/$ branching ratio & precision level & references \\
\hline$h_{i} \rightarrow \gamma \gamma, \gamma Z, g g$ & LO + NLO QCD & {$[102,104]$} \\
$h_{i} \rightarrow Z Z, W^{ \pm} W^{\mp}$ & reweighting of SM result & {$[105,106]$} \\
$h_{i} \rightarrow \bar{f} f$ & NLO & {$[107]$} \\
$H^{ \pm} \rightarrow f f^{\prime}$ & LO + NLO QCD & {$[108]$} \\
$h_{i} \rightarrow \widetilde{\chi}_{i}^{0} \widetilde{\chi}_{j}^{0}$ & LO & - \\
$h_{i} \rightarrow \widetilde{\chi}_{i}^{ \pm} \widetilde{\chi}_{j}^{\mp}$ & LO & - \\
$H^{ \pm} \rightarrow \widetilde{\chi}_{i}^{0} \widetilde{\chi}_{j}^{ \pm}$ & LO & - \\
$h_{i} \rightarrow h_{j} Z$ & LO & - \\
$H^{ \pm} \rightarrow h_{j} W^{ \pm}$ & LO & {$[108$} \\
$h_{i} \rightarrow h_{j} h_{k}$ & NLO + log resum. & {$[107,109$} \\
$h_{i} \rightarrow \tilde{f} \tilde{f}^{\prime}$ & LO & - \\
$H^{ \pm} \rightarrow \tilde{f}_{u} \tilde{f}_{d}^{\prime}$ & LO & {$[108$} \\
\hline
\end{tabular}

Table 1: Higgs decay widths/branching ratios computed by FeynHiggs. For decays including (excluding) loop corrections the $\mathbf{Z}$-matrix (U-matrix) is employed by default, which includes propagator-type corrections at the same level of accuracy as the mass predictions.

marized in Tab. 1 $\left.\right|^{3}$ Furthermore, approximations (for fast evaluation)-making use of tabulated SM results - of the main Higgs production cross-sections for given LHC energies and PDF sets are part of FeynHiggs, see Tab. 2.

Moreover, the output contains several electroweak precision observables (Tab. 3), flavour observables (Tab. 4), and a list of effective couplings. For many observables the corresponding SM predictions are given, in order to facilitate the comparison between MSSM and SM predictions. For the flavour observables, the recommendation is to use the values given in the output only to be added to the best available SM predictions (which are not provided by FeynHiggs), as in: $O_{\text {MSSM,best }}=$ $O_{\mathrm{SM}, \text { best }}+\left(O_{\mathrm{MSSM}, \mathrm{FH}}-O_{\mathrm{SM}, \mathrm{FH}}\right)$.

Note that the references listed in the Tables are not meant to provide a comprehensive literature list for the quoted observable. We list here only references containing corrections that are implemented into FeynHiggs.

\subsection{Using FeynHiggs}

FeynHiggs is mostly written in Fortran but can also be called from $\mathrm{C} / \mathrm{C}++$ and Mathematica, or accessed from a Web interface. In order to build FeynHiggs a Fortran

\footnotetext{
${ }^{3}$ Various refinements to some of these decays, discussed in [101, will soon be implemented in FeynHiggs.
} 


\begin{tabular}{|c|c|c|}
\hline production cross section & precision level & references \\
\hline $\bar{b} b \rightarrow h_{i}+X$ & reweighting of SM results & {$[86,87]$} \\
$\bar{b} b \rightarrow h_{i}+X$ (one tagged $\left.b\right)$ & reweighting of SM results & {$[86,87,110]$} \\
$g g \rightarrow h_{i}+X$ (c-factor) & reweighting of SM results & {$[86,87]$} \\
$g g \rightarrow h_{i}+X(\mathrm{k}$-factor) & reweighting of SM results & {$[86,87]$} \\
$q q \rightarrow q q h+X$ & reweighting of SM results & {$[86,87]$} \\
$q q, g g \rightarrow t \bar{t} h_{i}+X$ & reweighting of SM results & {$[86,87]$} \\
$q q \rightarrow W h_{i}+X$ & reweighting of SM results & {$[86,87]$} \\
$q q \rightarrow Z h_{i}+X$ & reweighting of SM results & {$[86,87]$} \\
$p p \rightarrow \tilde{t}_{1} \tilde{t}_{1} h$ & reweighting of SM results & {$[86,87]$} \\
$g b \rightarrow t H^{-}$ & reweighting of THDM results & {$[86,87,[11]$} \\
$t \rightarrow H^{+} b$ & LO + NLO QCD & {$[115,116]$} \\
\hline
\end{tabular}

Table 2: Higgs production cross-sections computed by FeynHiggs.

\begin{tabular}{|c|c|c|}
\hline EWPO & precision level & references \\
\hline$\Delta r$ & LO + NLO SUSY-QCD (full SM) & [46, 117, 119] \\
$\Delta \rho$ & NLO SUSY-QCD & [46, 117, 118] \\
$M_{W}$ & NLO + NNLO SUSY-QCD (full SM) & {$[46,117,119]$} \\
$\sin \theta_{W}^{\text {eff,lept }}$ & NLO + NNLO SUSY-QCD (full SM) & {$[46,117,120]$} \\
$g_{\mu}-2$ & LO + partial NLO & {$[121,122]$} \\
EDM of Th, n, and Hg & LO + partial NLO & {$[123,126]$} \\
\hline
\end{tabular}

Table 3: Electroweak precision observables computed by FeynHiggs. The abbreviation "full SM" is used to indicate that all known SM corrections are taken into account.

\begin{tabular}{|c|c|c|}
\hline flavour observable & precision level & references \\
\hline$B \rightarrow X_{s} \gamma$ & LO & {$[127$} \\
$\Delta M_{s}$ & LO + NLO QCD & {$[128$} \\
$B_{s} \rightarrow \mu^{+} \mu^{-}$ & LO + NLO QCD & {$[129$} \\
\hline
\end{tabular}

Table 4: flavour observables computed by FeynHiggs. All implemented corrections allow one to take non-minimal flavour violation into account. 
and $\mathrm{C}$ compiler and, to build the FeynHiggs executables for Mathematica, a working Mathematica/MathLink installation are needed. The code has been thoroughly tested with gfortran, ifort, and pgf90 in several versions on several platforms.

After downloading the latest tar file from http://feynhiggs.de, the configuration and installation follow these steps:

tar xvfz FeynHiggs-2.14.x.tar.gz

cd FeynHiggs-2.14.x

./configure

make

make install

After building the code, FeynHiggs provides several ways to use it:

- The FeynHiggs Fortran library libFH.a can be linked to Fortran or $\mathrm{C} / \mathrm{C}++$ programs, where the latter include CFeynHiggs.h.

- The FeynHiggs executable FeynHiggs allows one to run FeynHiggs from the command-line.

- The MathLink executable MFeynHiggs allows one to call FeynHiggs from within a Mathematica session.

The Web interface at http://feynhiggs.de/fhucc allows one to run FeynHiggs without downloading it.

For more details, we refer to the manual pages which are included in the tar file or are available at http://feynhiggs.de.

\section{Improvements of the fixed-order calculation}

In this section we describe improvements of the fixed-order calculation starting from FeynHiggs 2.13 (released in early 2017). The first improvement is the implementation of an optional $\overline{\mathrm{DR}}$ renormalization of the stop sector. Second, we discuss an adaptation of the renormalization in the Higgs sector at the two-loop level.

\subsection{Optional $\overline{D R}$ renormalization}

FeynHiggs by default employs a mixed OS $/ \overline{\mathrm{DR}}$ renormalization scheme (see [77] for more details). In particular, the parameters of the stop/top sector are defined using OS renormalization conditions [78] (stop masses and stop mixing parameter $X_{t}$ ) f] $^{4}$ FeynHiggs also offers the possibility to use $\overline{\mathrm{DR}}$ input parameters, however. Before the release of FeynHiggs 2.14, these were converted to OS parameters at the one-loop level.

\footnotetext{
${ }^{4}$ The counterterm of $X_{t}$ is fixed by imposing a condition on the off-diagonal stop mass counterterm $\delta m_{\tilde{t}_{1} \tilde{t}_{2}}$ employing on-shell external momenta. See e.g. [78] for more details.
} 

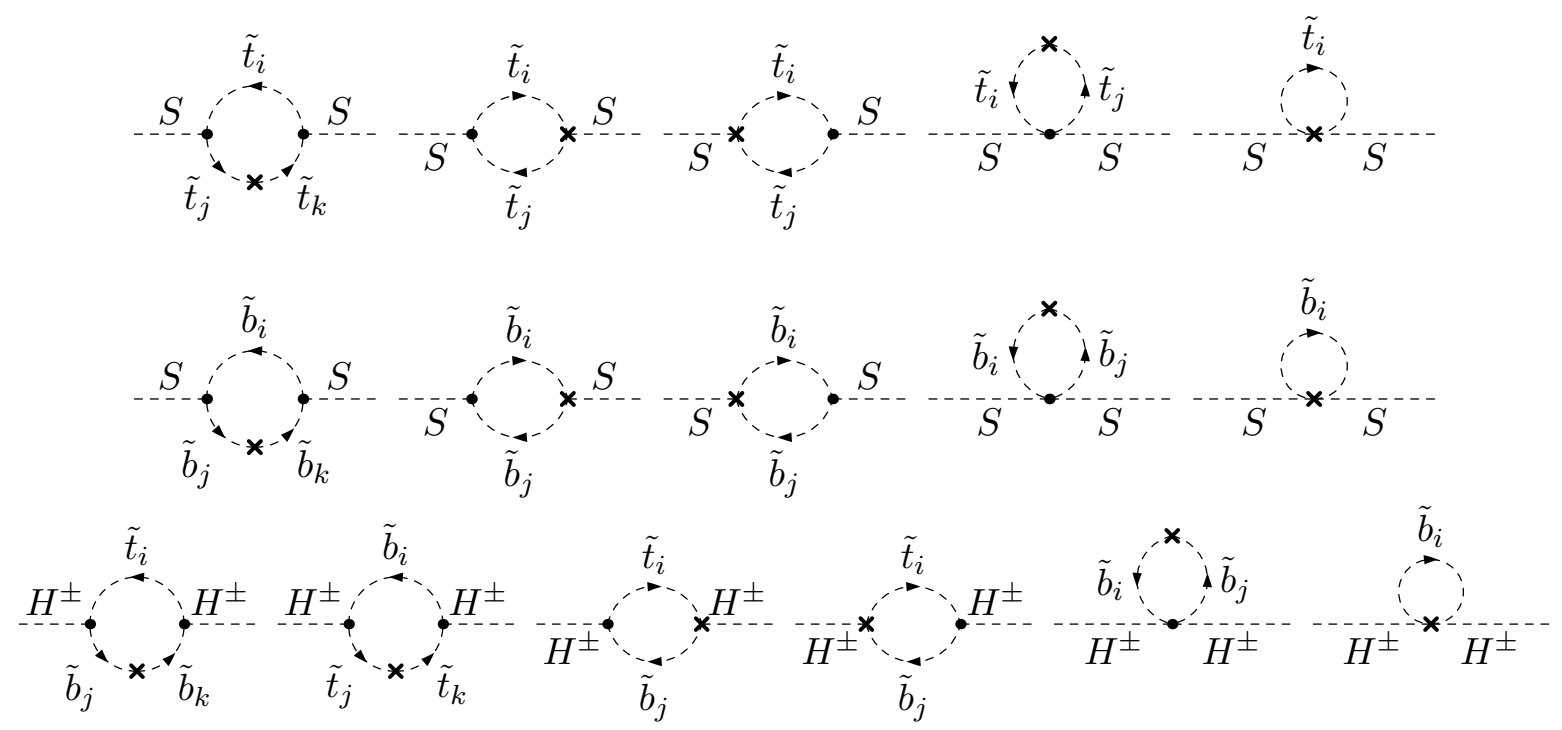

Figure 1: Generic two-loop subloop-renormalization diagrams appearing in the calculation of the $\overline{\mathrm{DR}}$ shifts $(S=h, H, A$ and $i, j, k=1,2)$. Due to the $S U(2)_{L}$ symmetry that relates the stop and sbottom sectors, also the diagrams containing only bottom squarks yield contributions involving stop counterterms.

The obtained OS parameters were then used as input for the rest of the calculation. This procedure has the advantage that a $\overline{\mathrm{DR}}$ result and the default $\mathrm{OS} / \overline{\mathrm{DR}}$ result of FeynHiggs can easily be compared. If the calculation is performed identically except for the renormalization schemes, the difference between the two results can be interpreted as a part of the theoretical uncertainty.

As shown in [63], this procedure is, however, problematic if the fixed-order result is supplemented by a resummation of large logarithms obtained in an EFT approach. The parameter conversion induces additional logarithmic higher-order terms which can become large for large SUSY scales and therefore spoil the resummation. To circumvent this issue, an optional $\overline{\mathrm{DR}}$ renormalization of the stop sector was employed in [63]. Here we describe the practical implementation of this optional renormalization scheme.

This scheme is implemented with the stop-mass scale $M_{S}=\sqrt{m_{\tilde{t}_{1}} m_{\tilde{t}_{2}}}$ as $\overline{\mathrm{DR}}$ scale. Inserting the relation ${ }^{5}$

$$
X_{t}^{\overline{\mathrm{DR}}}\left(M_{S}\right)=X_{t}^{\mathrm{OS}}+\left.\delta^{\mathrm{OS}} X_{t}\left(M_{S}\right)\right|_{\text {fin }}
$$

\footnotetext{
${ }^{5}$ The subscript "fin" indicates that only the finite part of the OS counterterm is taken into account. The UV-divergent part is cancelled by the corresponding $\overline{\mathrm{DR}}$ counterterm.
} 
and employing a Taylor expansion around $X_{t}^{\mathrm{OS}}$ we obtain

$$
\hat{\Sigma}\left(X_{t}^{\overline{\mathrm{DR}}}\left(M_{S}\right)\right)=\hat{\Sigma}\left(X_{t}^{\mathrm{OS}}\right)+\left.\left(\frac{\partial}{\partial X_{t}} \hat{\Sigma}\right) \cdot \delta^{\mathrm{OS}} X_{t}\left(M_{S}\right)\right|_{\text {fin }},
$$

where $\hat{\Sigma}$ is a generic renormalized self-energy, e.g. the $h h$ self-energy. The second term on the right-hand side corresponds to the subloop-renormalization diagrams involving $\delta X_{t}$ which are depicted in Fig. 1 .

In this way, changing the renormalization scheme and scale of the stop sector becomes straightforward $\sqrt{6}$ It amounts to the calculation of all subloop-renormalization diagrams involving the stop mass or the stop mixing counterterms with the renormalization scale set equal to the stop mass scale $M_{S}$. It should be noted that due to the $S U(2)_{L}$ gauge symmetry also some sbottom counterterms depend on stop counterterms (see e.g. [45]). Hence, also these contributions have to be taken into account. Adding the result to the existing self-energies with an OS renormalized stop sector, we have obtained the self-energies with a $\overline{\mathrm{DR}}$-renormalized stop sector.

This calculation is automated (see Section 5) and also works for complex input parameters. In contrast, the explicit conversion to OS parameters had been implemented for real input parameters only and was in practice applied to the absolute value while the phase was left unchanged. The new procedure is presently used in the stop sector of the mass calculation; if the parameters of the sbottom sector are input in the $\overline{\mathrm{DR}}$ scheme, FeynHiggs still uses the explicit $\overline{\mathrm{DR}} / \mathrm{OS}$ conversion to obtain the parameters renormalized in the mixed $\mathrm{OS} / \overline{\mathrm{DR}}$ scheme which is employed for the sbottom sector [35]. The explicit conversion is likewise still used for the calculation of other observables (e.g. decay rates to scalar tops), with the exception of the $h_{i} \rightarrow h_{j} h_{k}$ modes. For the calculation of the latter, the $\overline{\mathrm{DR}}$ parameters of the stop sector are used in order to consistently combine the NLO result [107, 109] with a resummation of large logarithms.

\subsection{Adapted renormalization of the Higgs sector}

Another improvement concerns the renormalization of the Higgs sector at the twoloop level. If the mass of the $\mathcal{C} \mathcal{P}$-odd Higgs boson $A$ is used as input mass (as done by default in the case of $(2 \times 2)$ mixing $)$, the following OS renormalization conditions are

\footnotetext{
${ }^{6}$ Another approach would have been to replace the on-shell counterterms by $\overline{\mathrm{DR}}$ counterterms taking into account the renormalization scale dependence.
} 
employed 7

$$
\begin{aligned}
\delta^{(1)} m_{A}^{2}= & \operatorname{Re}\left[\Sigma_{A A}^{(1)}\left(m_{A}^{2}\right)\right], \\
\delta^{(2)} m_{A}^{2}= & \operatorname{Re}\left[\Sigma_{A A}^{(2)}\left(m_{A}^{2}\right)\right]-\delta^{(1)} Z_{A A} \delta^{(1)} m_{A}^{2}-\delta^{(1)} Z_{G A} \delta^{(1)} m_{A G}^{2} \\
& +\operatorname{Im}\left[\Sigma_{A A}^{(1) \prime}\left(m_{A}^{2}\right)\right] \operatorname{Im}\left[\Sigma_{A A}^{(1)}\left(m_{A}^{2}\right)\right],
\end{aligned}
$$

where the $\delta^{(1)} Z$-s are one-loop field renormalization constants (following the conventions of [80]).

The physical mass squared, $M_{A}^{2}$, is given by the real part of the corresponding propagator pole. In the absence of $\mathcal{C} \mathcal{P}$-violation, i.e. if all input parameters are real, this pole is obtained by solving the equation

$$
p^{2}-m_{A}^{2}+\hat{\Sigma}_{A A}\left(p^{2}\right)=0 .
$$

Expanding up to the two-loop level yields

$$
M_{A}^{2}=m_{A}^{2}-\operatorname{Re}\left[\hat{\Sigma}_{A A}^{(1)}\left(m_{A}^{2}\right)\right]-\operatorname{Re}\left[\hat{\Sigma}_{A A}^{(2)}\left(m_{A}^{2}\right)\right]+\operatorname{Re}\left[\hat{\Sigma}_{A A}^{(1) \prime}\left(m_{A}^{2}\right) \hat{\Sigma}_{A A}^{(1)}\left(m_{A}^{2}\right)\right]
$$

where the renormalized self-energies, marked by a hat, are given in terms of the unrenormalized self-energies containing the subloop renormalization and counterterms by

$$
\begin{aligned}
& \hat{\Sigma}_{A A}^{(1)}\left(m_{A}^{2}\right)=\Sigma_{A A}^{(1)}\left(m_{A}^{2}\right)-\delta^{(1)} m_{A}^{2} \\
& \hat{\Sigma}_{A A}^{(2)}\left(m_{A}^{2}\right)=\Sigma_{A A}^{(2)}\left(m_{A}^{2}\right)-\delta^{(1)} Z_{A A} \delta^{(1)} m_{A}^{2}-\delta^{(1)} Z_{A G} \delta^{(1)} m_{A G}^{2}-\delta^{(2)} m_{A}^{2} .
\end{aligned}
$$

The superscript marks the loop order, and the prime is used to denote a derivative with respect to $p^{2}$. Employing the conditions defined in Eqs. (4) and (5), we straightforwardly obtain

$$
M_{A}^{2}=m_{A}^{2}
$$

meaning that the input mass $m_{A}$ is equivalent to the physical mass $M_{A}$. Before the release of FeynHiggs 2.14.0, the term in the last line of Eq. (5) had been omitted.

If the charged Higgs boson mass $m_{H^{ \pm}}$is used as input parameter and renormalized on-shell (as done by default in the case of $(3 \times 3)$ mixing in the neutral Higgs sector),

\footnotetext{
${ }^{7}$ At the two-loop level, all self-energy contributions implemented in FeynHiggs are obtained by default in the limit of vanishing external momentum. Therefore, the counterterms are adapted accordingly if they appear at the two-loop level (see e.g. [80] for more details). An exception are the $\mathcal{O}\left(\alpha_{t} \alpha_{s}\right)$ corrections for which optionally the full momentum dependence can be taken into account [54,58]. Note that the new additional contribution to the two-loop counterterms $\delta^{(2)} m_{A}^{2}$ and $\delta^{(2)} m_{H^{ \pm}}^{2}$, discussed in this section, is not of $\mathcal{O}\left(\alpha_{t} \alpha_{s}\right)$.
} 
its two-loop counterterm is adapted accordingly,

$$
\begin{aligned}
\delta^{(2)} m_{H^{ \pm}}^{2}= & \operatorname{Re}\left[\Sigma_{H^{ \pm} H^{ \pm}}^{(2)}\left(m_{H^{ \pm}}^{2}\right)\right]-\delta^{(1)} Z_{H^{ \pm} H^{ \pm}} \delta^{(1)} m_{H^{ \pm}}^{2} \\
& -\frac{1}{2}\left(\delta^{(1)} Z_{G^{ \pm} H^{ \pm}} \delta^{(1)} m_{H^{ \pm} G^{ \pm}}+\delta^{(1)} Z_{G^{ \pm} H^{ \pm}}^{*} \delta^{(1)} m_{G^{ \pm} H^{ \pm}}\right) \\
& +\operatorname{Im}\left[\Sigma_{H^{ \pm} H^{ \pm}}^{(1) \prime}\left(m_{H^{ \pm}}^{2}\right)\right] \operatorname{Im}\left[\Sigma_{H^{ \pm} H^{ \pm}}^{(1)}\left(m_{H^{ \pm}}^{2}\right)\right],
\end{aligned}
$$

whereas

$$
\delta^{(2)} m_{A}^{2}=\delta^{(2)} m_{H^{ \pm}}^{2}-\delta^{(2)} M_{W}^{2}
$$

In the approximation of vanishing electroweak gauge couplings, as employed for all twoloop corrections implemented in FeynHiggs, the two-loop counterterm of the $W$ boson mass $\delta^{(2)} M_{W}^{2}$ is equal to zero.

\section{Improvements of the EFT calculation}

Apart from the fixed-order calculation, also the EFT calculation that is implemented in FeynHiggs has been improved.

The first advancement concerns the threshold corrections. Up to FeynHiggs 2.12.2, all threshold corrections were implemented in their degenerate form. This means that at a threshold all particles which are integrated out were assumed to have the same mass, which is moreover equal to the matching scale. As an example, in the EFT calculation it was assumed that the soft SUSY-breaking masses of the stop sector, $M_{Q_{3}}$ and $M_{U_{3}}$, are equal to each other. No such assumptions have been made in the fixed-order calculation, however. Therefore, the effect of non-degeneracy was captured completely at the oneloop level and at the two-loop level in the limit of vanishing electroweak gauge couplings via the diagrammatic part of the hybrid calculation.

In FeynHiggs 2.13.0, the full non-degenerate one-loop and the two-loop threshold corrections of $\mathcal{O}\left(\alpha_{t} \alpha_{s}\right)$ [55] have been implemented. In FeynHiggs 2.14.1, also the nondegenerate two-loop threshold corrections of $\mathcal{O}\left(\alpha_{t}^{2}\right)$ [62] have been included.

This facilitated to lift also a further restriction. Before, the low-energy threshold of the gluino could only be taken into account in the case of LL and NLL resummation. If NNLL resummation was activated, the gluino mass $M_{\tilde{g}}$ was set equal to the SUSY scale $M_{\text {SUSY }}$ in the EFT calculation. The implementation of the non-degenerate threshold correction of $\mathcal{O}\left(\alpha_{t} \alpha_{s}\right)$ enables us to set $M_{\tilde{g}}$ independently of $M_{\mathrm{SUSY}}$ in all relevant 
threshold corrections. ${ }^{8}$ No two-loop threshold corrections need to be taken into account if the gluino is integrated out from the effective theory below the scale $M_{\mathrm{SUSY}}$. This is due to the couplings of the gluino: It couples either through a quark-squark-gluino or a gluon-gluino-gluino vertex. Therefore, the gluino only contributes to the matching of the Higgs self-coupling in the effective theory below the scale $M_{\text {SUSY }}$-in which all squarks are integrated out - at the three-loop level and beyond. The RGEs of the EFT are modified already at the one-loop level, however. Corresponding one- and twoloop RGEs are listed in [60. The modifications of the three-loop RGEs are unknown. Based on the finding that in the SM the effects from three-loop running are negligible (see e.g. [59]), it is conceivable that also the three-loop running in the SM plus gluino is negligible.

As second improvement, an interpolation of the EFT result was introduced for complex parameters in FeynHiggs 2.13.0. A pure EFT calculation taking into account phases in the threshold corrections has been performed in Ref. [130], however, for the hybrid approach no calculation for complex parameters is available at the moment. Therefore, we follow the approach that is employed in FeynHiggs for those fixed-order contributions which are only known for the case of real parameters and interpolate the EFT calculation in the case of complex parameters. The interpolation is carried out for the Higgsino mass parameter $\mu$, the trilinear coupling in the stop sector $A_{t}$, and the gluino mass parameter $M_{3}$, which are all allowed to take complex values. The interpolation is performed by evaluating the EFT result at $|P|$ and $-|P|\left(P=\mu, A_{t}, M_{3}\right)$ and afterwards linearly interpolating between the obtained values.

\section{Improvements of code structure}

The code of FeynHiggs is structured in three parts:

- code hand-written for FeynHiggs

The 'back bone' of FeynHiggs is of course written by hand. Most code has been developed specifically for FeynHiggs, with some adaptations from external sources, e.g. LoopTools [131] or SLHALib [132]. Code falling into this category includes

- all structural code: data structures, frontend, I/O, record handling, etc.

- utility functions: matrix diagonalization, loop integrals, ordinary-differentialequation solver, etc.

- contributions taken from the literature: the EDMs, some of the RGEs and threshold corrections in the EFT sector, higher-order SM parts of $\Delta r$, etc.

\footnotetext{
${ }^{8}$ Note that formally we would have to expand the threshold corrections in $M_{\tilde{g}} / M_{\text {SUSY }}$ if the gluino remains in the EFT below the scale $M_{\mathrm{SUSY}}$. However, from a practical point of view contributions of $\mathcal{O}\left(M_{\tilde{g}} / M_{\mathrm{SUSY}}\right)$ in the treshold corrections are negligible in scenarios in which the gluino threshold has a sizeable numerical impact (i.e. if $M_{\tilde{g}} \ll M_{\mathrm{SUSY}}$ ). It should be noted that logarithms involving the SUSY scale and the gluino mass are not resummed for $M_{\tilde{g}}>M_{\mathrm{SUSY}}$.
} 
- code generated from external expressions

FeynHiggs includes several contributions which originated from independent projects and for which the original (typically large) expressions are available, usually in Mathematica format: several of the two-loop contributions to the Higgs selfenergies, several ingredients of the EFT calculation, the muon $g-2$, the two-loop parts of $\Delta r$, etc.

This is already more practical than hand-coded expressions since modifications (e.g. a change in conventions) can be done in Mathematica which is much easier and safer than search/replace in an editor. Also the code can be re-generated at any time and can be optimized, too. On the other hand, it is nearly impossible to extend or significantly change results implemented in this way.

- code generated from calculations done in/for FeynHiggs

This mode is most convenient for perturbatively calculable quantities since it allows full control over model content, particle selection, resummations $/ K$-factors, the renormalization prescription, etc. Calculations done in this way can usually be generalized to other models relatively straightforwardly. Note that we do not pursue a 'generator generator' approach as done in some other packages, i.e. even if our scripts ran (or were modified to run) with an 'arbitrary' model file, the produced code would still need to be embedded in and called from the main program, in which the inputs have to be properly adjusted.

Calculations at this stage of automation can be found in the 'gen' subdirectory of FeynHiggs and currently include

- the entire set of renormalized one-loop Higgs self-energies (gen/oneloop),

- the $\mathcal{O}\left(\alpha_{t}^{2}\right)$ contributions to the two-loop Higgs self-energies (gen/tlsp),

- the shifts at two-loop order from $\overline{\mathrm{DR}}$ input parameters (gen/drbar),

- the shifts at two-loop order from finite $Z$ factors (gen/dzhfin),

- the one-loop decay rates (gen/decays),

- the one-loop corrections to $\Delta_{b}$ (gen/db),

- several flavour observables at the one-loop order (gen/bsg, bsll, dms),

- the one-loop MSSM contribution to $\Delta \rho$ (gen/deltarho)

The code-generation scripts generally follow the approach of [90] and, in case of improvements or bugfixes, can be re-run with a few keystrokes.

(Another subdirectory, 'gen/prod', contains code for the empirical fitting of crosssections from tabulated data. It falls somewhat outside the sort of code generation described here and shall not be discussed further.) 
In the following we describe the main improvements in FeynHiggs version 2.14.

The unrenormalized one-loop Higgs self-energies have been generated with a high degree of automatization for all versions since 2.0. Before FeynHiggs 2.14, however, the entire renormalization was hard-coded. (At the time of the first implementation of the self-energies, a model file for the MSSM including the complete set of one-loop counter-terms [133] did not yet exist.) Old FeynHiggs versions actually encoded various options of renormalization schemes which were used for testing at that time. The only recommended scheme became the one used in the model file. The flags fieldren and tanbren, which selected these schemes, were correspondingly dropped in 2.14.

The new procedure instead reads the renormalization (counter-terms plus renormalization constants) from the model file, making as few assumptions as possible. It needs to know the relevant flags governing the renormalization, of course, such as \$MHpInput, which selects whether $M_{A}$ or $M_{H^{+}}$is the input mass for the Higgs sector, for which it generates the necessary if statements in the output. Diagram computation and code generation rely heavily on FeynArts [134] and FormCalc [131], and to achieve the level of automation we desired, we had to enhance and add several of FormCalc's code-generation functions.

New in 2.14 are also the two-loop shifts induced by the use of $\overline{\mathrm{DR}}$ input parameters and finite field-renormalization factors in the one-loop Higgs self-energies.

Even though FeynHiggs does not (yet) go beyond the MSSM in scope, there are three 'models' used internally: 'mfv' and 'nmfv', the MSSM with minimal and nonminimal flavour-violation, and ' $g l$ ', the gaugeless version used e.g. in the two-loop calculations. An important task was also to consolidate various sources of Feynman rules for the MSSM which had grown over the years.

All one-loop self-energies are automatically split into the parts corresponding to FeynHiggs' mssmpart flag: $t / \tilde{t} ; t / \tilde{t}+b / \tilde{b} ; f / \tilde{f}$; all, so that individual sectors of the MSSM can still be looked at even in the presence of a generated renormalization. Our code generation routines are generic enough to deal with things such as different renormalization schemes and simple extensions of the MSSM but are also to a certain extent modelaware, e.g. know how to simplify the $(2 \times 2)$ sfermion mixing matrices, and are hence not directly applicable to 'arbitrary' models. Planned directions in this programme are the implementation of recent two-loop results (e.g. [82, 83]) and the extension to the NMSSM based on [98, 101, 135].

Finally, the adherence to the FORTRAN 77 standard, kept mainly because of g77 (for many years the only free Fortran compiler), was dropped with version 2.14. Even though outwardly the code retains its fixed-format 'F77' look, it uses many F90 idioms, in particular vector syntax.

While the numerical stability of the code is generally satisfactory, some sections, for example the non-degenerate two-loop threshold corrections of the EFT results, can be affected by numerical artefacts even in not-too-extreme corners of the parameter space. A quadruple-precision version of FeynHiggs has been available for some time (./configure --quad) but this naturally runs vastly slower. In 2.14.3 we reorganized 

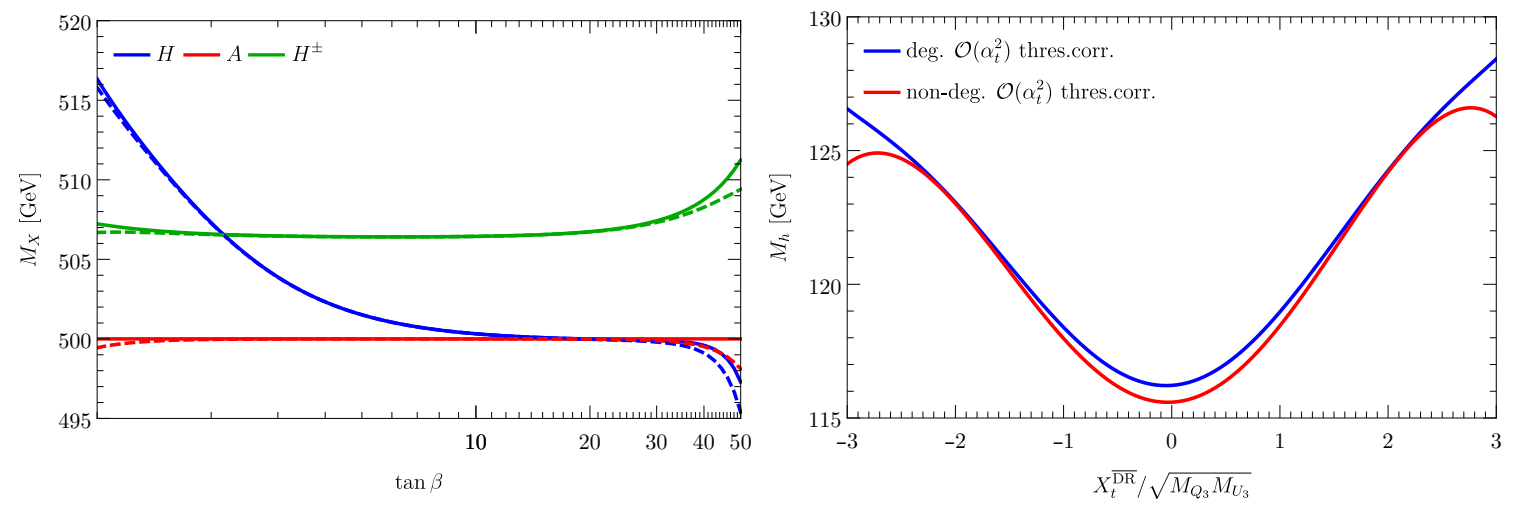

Figure 2: Left: Masses of the non-SM-like Higgs bosons as a function of $\tan \beta$. The results employing the adapted renormalization of the Higgs sector (solid) are compared to the results employing the old renormalization (dashed). Right: $M_{h}$ as a function of $X_{t}^{\overline{\mathrm{DR}}} / \sqrt{M_{Q_{3}} M_{U_{3}}}$. The results obtained using the non-degenerate and the degenerate form of the threshold correction of $\mathcal{O}\left(\alpha_{t}^{2}\right)$ are compared. (See text for the values of the parameters.)

many of the internal utility functions, in particular the loop integrals, so that they compile to either a double- or a quadruple-precision object depending on the setting of a flag, and can now adjust higher precision for just the neuralgic parts, which improves overall precision appreciably and makes the slowdown hardly noticeable. Quadruple precision (REAL*16, COMPLEX $* 32)$ is currently available with gfortran and ifort. With gfortran, the alternate extended-precision type $\mathrm{REAL} * 10$ can also be targeted, which is realized in hardware on Intel x86 chips, either overall (./configure --quad --real10) or just for the parts in need of extra precision (./configure --real10).

\section{Numerical results}

In this Section, we present some exemplary results highlighting various aspects of the improvements discussed above. Other examples of the improved Higgs-boson mass calculation are given in [63, 67, 136, 137].

\subsection{Improvements of the fixed-order calculation}

First, we look at the improvements of the fixed-order calculation as dissused in Section 3 the numerical impact of the new optional $\overline{\mathrm{DR}}$ renormalization on $M_{h}$ obtained as a result of the hybrid approach has already been presented in [63], and we do not repeat this discussion here. We will, however, investigate scenarios with complex $\overline{\mathrm{DR}}$ input parameters in Section 6.2.

The numerical effect of the adapted renormalization of the Higgs sector, see Section 3. namely of the additional term $\operatorname{Im}\left[\Sigma^{(1) \prime}\right] \operatorname{Im}\left[\Sigma^{(1)}\right]$ in the two-loop counterterm of the input mass in Eq. (5) or Eq. (11) is shown in the left plot of Fig. 2 for a scenario 
with the input values $M_{\mathrm{SUSY}}=1 \mathrm{TeV}$ (common mass scale of squarks and sleptons), $X_{t}^{\mathrm{OS}} / M_{\mathrm{SUSY}}=2, m_{A}=500 \mathrm{GeV}$, and $\mu=-500 \mathrm{GeV}$. The gaugino masses are set to $M_{1}=M_{2}=500 \mathrm{GeV}$, and $M_{3}=2.5 \mathrm{TeV}$. All trilinear soft-breaking couplings apart from $A_{t}$ are set to zero.

Due to the chosen mass pattern, the additional term $\operatorname{Im}\left[\Sigma_{A A}^{(1) \prime}\left(m_{A}^{2}\right)\right] \operatorname{Im}\left[\Sigma_{A A}^{(1)}\left(m_{A}^{2}\right)\right]$ only receives contributions from SM particles. One observes that the term is negligible in the range $2 \lesssim \tan \beta \lesssim 25$. For $\tan \beta \sim 1$, where the coupling of the heavy Higgs bosons to top quarks is not suppressed, a small upward shift of all three non-SMlike Higgs-boson masses is visible. Similarly, one finds a slightly larger upward shift for $\tan \beta \gtrsim 25$, where the coupling of the heavy Higgs bosons to bottom quarks becomes large. One also observes that with the adapted renormalization scheme the physical mass of the $A$-boson is, as expected, always equal to the input mass $m_{A}$.

\subsection{Improvements of the EFT calculation}

Next, we discuss the numerical impact of the improvements of the EFT calculation. We first consider the effect of the non-degenerate threshold corrections. Since, as already mentioned, the effect of non-degenerate particle masses was captured exactly up to the level of two-loop corrections via the fixed-order calculation before, the numerical impact of those for scenarios with SUSY masses around the TeV scale is quite small $(\lesssim \mathcal{O}(100 \mathrm{MeV}))$.

For multi-TeV SUSY masses larger effects can be observed, however. As an example, we investigate a scenario in which all soft-breaking masses, the mass of the $\mathcal{C} \mathcal{P}$-odd Higgs boson, $m_{A}$, and the Higgsino mass parameter $\mu$ are set equal to $M_{\mathrm{SUSY}}=5 \mathrm{TeV}$. Only the soft-breaking mass $M_{U_{3}}$ in the stop sector is chosen differently, $M_{U_{3}}=M_{\mathrm{SUSY}} / 4$, to generate a large non-degeneracy in the stop sector. $\tan \beta$ is set equal to 10 . In the right plot of Fig. 2, we show $M_{h}$ as a function of $X_{t}^{\overline{\mathrm{DR}}} / \sqrt{M_{Q_{3}} M_{U_{3}}}$, comparing the results obtained with the degenerate and the non-degenerate threshold corrections of $\mathcal{O}\left(\alpha_{t}^{2}\right)$. Due to the multi-TeV SUSY scale we observe a downwards shift of $\sim 1 \mathrm{GeV}$ for vanishing stop mixing. Moreover, we see that the values of $X_{t}^{\overline{\mathrm{DR}}}$ maximizing $M_{h}$ are shifted away from the expected value of $\left|X_{t}^{\overline{\mathrm{DR}}} / \sqrt{M_{Q_{3}} M_{U_{3}}}\right| \sim \sqrt{6}$ if the degenerate threshold correction of $\mathcal{O}\left(\alpha_{t}^{2}\right)$ is used. This effect was especially relevant for the studies conducted in [137.

For a further example showing the impact of the non-degenerate threshold corrections of $\mathcal{O}\left(\alpha_{t}^{2}\right)$, we refer to [67] where scenarios with low $m_{A}$ are investigated and shifts of up to $6 \mathrm{GeV}$ have been found between results obtained using the degenerate and non-degenerate threshold corrections of $\mathcal{O}\left(\alpha_{t}^{2}\right)$.

As second improvement we investigate the interpolation of the EFT result for the case of complex input parameters. We compare three methods to handle complex parameters in the EFT calculation: using the real part of the complex parameter as input, using its absolute value as input, and the interpolation method described in Section 4. For the investigation, we use a scenario like the one in the right plot of 

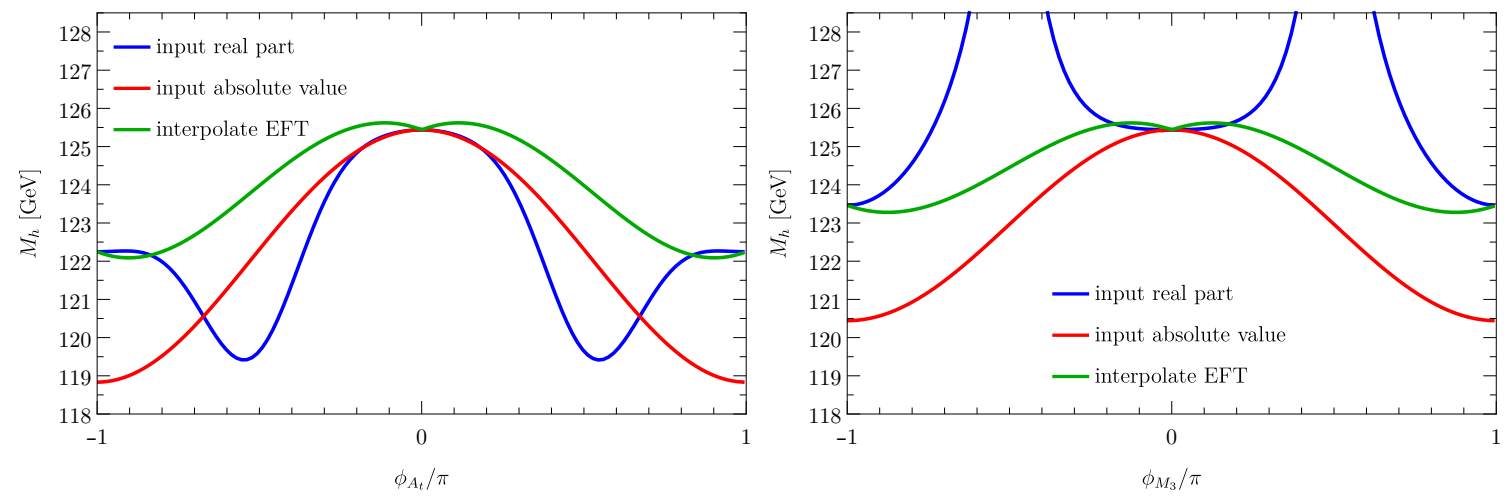

Figure 3: Comparison of results with and without interpolation of the EFT result for complex parameters. The input parameters $M_{\mathrm{SUSY}}=2 \mathrm{TeV}, \tan \beta=10$ and $X_{t}^{\overline{\mathrm{DR}}} / M_{\mathrm{SUSY}}=\sqrt{6}$ are chosen. Left: $M_{h}$ as a function of $\phi_{A_{t}}$. Right: $M_{h}$ as a function of $\phi_{M_{3}}$.

Fig. 2 but with $M_{U_{3}}=M_{\text {SUSY }}=2 \mathrm{TeV}$. In addition, we allow for nonzero phases of $A_{t}$ and $M_{3}$.

In the left panel of Fig. 3 we vary the phase of $A_{t}$ between $-\pi$ and $\pi$ and observe shifts in $M_{h}$ of up to $3 \mathrm{GeV}$ for $\phi_{A_{t}} \sim \pm \frac{\pi}{4}$. Cutting off the imaginary part of $A_{t}$ leads to values of $M_{h}$ which are similar to those obtained from the interpolation in $\phi_{A_{t}}$ only close to $\phi_{A_{t}}=0$; $\pm \pi$ where the imaginary part of $A_{t}$ is small. For phases in between, the predicted values of $M_{h}$ are smaller compared to those obtained from the interpolation. Using the absolute value conversely works better for $\left|\phi_{A_{t}}\right| \lesssim 0.7$ but is worse, as expected, for $\phi_{A_{t}} \sim \pm \pi$. Since the one-loop threshold correction involves only even powers of $X_{t}$, and in the investigated scenario $A_{t}$ is similar in size to $X_{t}$ due to the relatively high value of $\tan \beta$, the dominant contribution causing these shifts is the threshold correction of $\mathcal{O}\left(\alpha_{t} \alpha_{s}\right)$.

This is confirmed by the right plot of Fig. 3, showing a variation of the gluino phase $\phi_{M_{3}}$. The threshold correction of $\mathcal{O}\left(\alpha_{t} \alpha_{s}\right)$ is a function of $X_{t} / M_{3}$. Therefore, a variation of $\phi_{M_{3}}$ is comparable to a variation of $\phi_{A_{t}}$, as observable in the plots. Cutting off the imaginary part of $M_{3}$ is not a good approximation here since $M_{3}$ appears in the denominator and its real part approaches zero for $\phi_{M_{3}} \sim \pm \frac{\pi}{4}$.

Given that the different treatment of the phases is formally of three-loop order (at the one- and two-loop level the phase dependence is taken into account without approximation) the effects are remarkably large. In contrast, a variation of $\phi_{\mu}$ leads only to very small shifts well below $1 \mathrm{GeV}$.

The plots shown in Fig. 3 are also examples of scenarios with complex $\overline{\mathrm{DR}}$ input parameters. The conversion between the $\overline{\mathrm{DR}}$ input parameters and the internally used OS parameters, as employed in earlier FeynHiggs versions, was in contrast not applicable to the case of complex parameters (i.e. the phases were not converted to the OS scheme). 


\section{Conclusions}

After presenting a short overview over the Fortran code FeynHiggs (available at http://feynhiggs.de), whose main purpose is to provide precise numerical predictions for observables in the Higgs sector of the MSSM, we discussed various improvements in the calculation of the Higgs spectrum. For the prediction of the Higgs-boson masses, a diagrammatic fixed-order calculation - accurate for low SUSY scales - is combined with an EFT calculation - accurate for high SUSY scales - in order to provide an accurate result also for intermediate scales.

We first discussed improvements of the fixed-order calculation. We explained the implementation of an alternative $\overline{\mathrm{DR}}$ renormalization of the stop sector (allowing one to input also complex $\overline{\mathrm{DR}}$ parameters). Moreover, we showed how the two-loop renormalization of the Higgs sector is adapted in order to ensure that the input Higgs mass is equal to the corresponding physical mass. Numerically, this change of the renormalization scheme has been relevant in the considered scenario only for very low or very high values of $\tan \beta$.

Then, we addressed the improvements of the EFT calculation. We described the implementation of threshold corrections valid for arbitrary masses of the decoupled particles and showed that this can lead to sizeable numerical effects as compared to the result using degenerate threshold corrections e.g. in the case of a large separation between the two stop masses. Moreover, we explained how the EFT calculation is interpolated in the case of complex input parameters. The numerical effects of the phase variations can be important if the imaginary parts of the respective parameters are sufficiently large.

We furthermore highlighted several improvements of the code structure, which are not directly visible for the user but should allow for an easier development and extension of FeynHiggs in the future.

\section{Acknowledgments}

We thank E. Bagnaschi, P. Slavich, and I. Sobolev for useful discussions and relentless testing. We thank E. Bagnaschi, P. Slavich, D. Stöckinger, K. Williams, and L. Zeune for contributions to the code. The work of S.H. is supported in part by the MEINCOP Spain under contract FPA2016-78022-P, in part by the "Spanish Agencia Estatal de Investigación" (AEI) and the EU "Fondo Europeo de Desarrollo Regional" (FEDER) through the project FPA2016-78022-P, in part by the "Spanish Red Consolider MultiDark" FPA2017-90566-REDC, and in part by the AEI through the grant IFT Centro de Excelencia Severo Ochoa SEV-2016-0597. G.W. acknowledges support by the DFG through the SFB 676 "Particles, Strings and the Early Universe". The work of S.P. is supported by the ANR grant "HiggsAutomator" (ANR-15-CE31-0002). HR's work is partially funded by the Danish National Research Foundation, grant number DNRF90. The authors would like to express special thanks to the Mainz Institute for Theoretical Physics (MITP) for its hospitality and support. 


\section{References}

[1] G. Aad, et al., Observation of a new particle in the search for the Standard Model Higgs boson with the ATLAS detector at the LHC, Phys. Lett. B716 (2012) 1-29. arXiv:1207.7214, doi: 10.1016/j physletb.2012.08.020. [p 4]

[2] S. Chatrchyan, et al., Observation of a new boson at a mass of $125 \mathrm{GeV}$ with the CMS experiment at the LHC, Phys. Lett. B716 (2012) 30-61. arXiv:1207.7235, doi:10.1016/j.physletb. 2012.08.021. [p 4]

[3] G. Aad, et al., Combined Measurement of the Higgs Boson Mass in $p p$ Collisions at $\sqrt{s}=7$ and $8 \mathrm{TeV}$ with the ATLAS and CMS Experiments, Phys. Rev. Lett. 114 (2015) 191803. arXiv: 1503.07589, doi:10.1103/PhysRevLett.114.191803.

[4] G. Aad, et al., Measurements of the Higgs boson production and decay rates and constraints on its couplings from a combined ATLAS and CMS analysis of the LHC pp collision data at $\sqrt{s}=7$ and $8 \mathrm{TeV}$, JHEP 08 (2016) 045. arXiv:1606.02266, doi:10.1007/JHEP08(2016)045. [p4

[5] H. P. Nilles, Supersymmetry, Supergravity and Particle Physics, Phys. Rept. 110 (1984) 1-162. doi:10.1016/0370-1573(84)90008-5. [p 4]

[6] H. E. Haber, G. L. Kane, The Search for Supersymmetry: Probing Physics Beyond the Standard Model, Phys. Rept. 117 (1985) 75-263. doi:10.1016/0370-1573(85)90051-1. [p 4

[7] J. R. Ellis, G. Ridolfi, F. Zwirner, Radiative corrections to the masses of supersymmetric Higgs bosons, Phys. Lett. B257 (1991) 83-91. doi:10.1016/0370-2693(91) 90863-L. [p 4]

[8] Y. Okada, M. Yamaguchi, T. Yanagida, Upper bound of the lightest Higgs boson mass in the minimal supersymmetric standard model, Prog. Theor. Phys. 85 (1991) 1-6. doi:10.1143/ptp/ 85.1.1.

[9] Y. Okada, M. Yamaguchi, T. Yanagida, Renormalization group analysis on the Higgs mass in the softly broken supersymmetric standard model, Phys. Lett. B262 (1991) 54-58. doi: 10.1016/0370-2693(91) 90642-4.

[10] H. E. Haber, R. Hempfling, Can the mass of the lightest Higgs boson of the minimal supersymmetric model be larger than $\mathrm{m}(\mathrm{Z})$ ?, Phys. Rev. Lett. 66 (1991) 1815-1818. doi: 10.1103/PhysRevLett.66.1815.

[11] J. R. Ellis, G. Ridolfi, F. Zwirner, On radiative corrections to supersymmetric Higgs boson masses and their implications for LEP searches, Phys. Lett. B262 (1991) 477-484. doi:10. 1016/0370-2693(91)90626-2.

[12] K. Sasaki, M. Carena, C. E. M. Wagner, Renormalization group analysis of the Higgs sector in the minimal supersymmetric standard model, Nucl. Phys. B381 (1992) 66-86. doi:10.1016/ 0550-3213(92) 90640-W.

[13] P. H. Chankowski, S. Pokorski, J. Rosiek, Charged and neutral supersymmetric Higgs boson masses: Complete one loop analysis, Phys. Lett. B274 (1992) 191-198. doi:10.1016/ 0370-2693(92) 90522-6.

[14] A. Brignole, Radiative corrections to the supersymmetric neutral Higgs boson masses, Phys. Lett. B281 (1992) 284-294. doi:10.1016/0370-2693(92)91142-V.

[15] P. H. Chankowski, S. Pokorski, J. Rosiek, Complete on-shell renormalization scheme for the minimal supersymmetric Higgs sector, Nucl. Phys. B423 (1994) 437-496. arXiv:hep-ph/9303309, doi:10.1016/0550-3213(94)90141-4. [pp 5.6.6.

[16] R. Hempfling, A. H. Hoang, Two loop radiative corrections to the upper limit of the lightest Higgs boson mass in the minimal supersymmetric model, Phys. Lett. B331 (1994) 99-106. arXiv: hep-ph/9401219, doi:10.1016/0370-2693(94)90948-2.

[17] J. A. Casas, J. R. Espinosa, M. Quiros, A. Riotto, The lightest Higgs boson mass in the minimal supersymmetric standard model, Nucl. Phys. B436 (1995) 3-29, [Erratum: Nucl. Phys.B439,466(1995)]. arXiv:hep-ph/9407389, doi:10.1016/0550-3213(94)00508-C, 10 . 1016/0550-3213(95) 00057-Y.

[18] A. Dabelstein, The one loop renormalization of the MSSM Higgs sector and its application 
to the neutral scalar Higgs masses, Z. Phys. C67 (1995) 495-512. arXiv:hep-ph/9409375, doi:10.1007/BF01624592. [p 5]

[19] M. Carena, J. R. Espinosa, M. Quiros, C. E. M. Wagner, Analytical expressions for radiatively corrected Higgs masses and couplings in the MSSM, Phys. Lett. B355 (1995) 209-221. arXiv: hep-ph/9504316, doi:10.1016/0370-2693(95)00694-G.

[20] M. Carena, M. Quiros, C. E. M. Wagner, Effective potential methods and the Higgs mass spectrum in the MSSM, Nucl. Phys. B461 (1996) 407-436. arXiv:hep-ph/9508343, doi:10. 1016/0550-3213(95)00665-6.

[21] D. M. Pierce, J. A. Bagger, K. T. Matchev, R.-J. Zhang, Precision corrections in the minimal supersymmetric standard model, Nucl. Phys. B491 (1997) 3-67. arXiv:hep-ph/9606211, doi: 10.1016/S0550-3213(96)00683-9. [p 5

[22] H. E. Haber, R. Hempfling, A. H. Hoang, Approximating the radiatively corrected Higgs mass in the minimal supersymmetric model, Z. Phys. C75 (1997) 539-554. arXiv:hep-ph/9609331. doi: $10.1007 / \mathrm{s} 002880050498$

[23] S. Heinemeyer, W. Hollik, G. Weiglein, QCD corrections to the masses of the neutral CPeven Higgs bosons in the MSSM, Phys. Rev. D58 (1998) 091701. arXiv:hep-ph/9803277, doi:10.1103/PhysRevD.58.091701

[24] S. Heinemeyer, W. Hollik, G. Weiglein, Precise prediction for the mass of the lightest Higgs boson in the MSSM, Phys. Lett. B440 (1998) 296-304. arXiv:hep-ph/9807423, doi:10.1016/ S0370-2693(98)01116-2.

[25] R.-J. Zhang, Two loop effective potential calculation of the lightest CP even Higgs boson mass in the MSSM, Phys. Lett. B447 (1999) 89-97. arXiv:hep-ph/9808299, doi:10.1016/ S0370-2693(98)01575-5.

[26] S. Heinemeyer, W. Hollik, G. Weiglein, The masses of the neutral CP-even Higgs bosons in the MSSM: Accurate analysis at the two loop level, Eur. Phys. J. C9 (1999) 343-366. arXiv: hep-ph/9812472, doi:10.1007/s100529900006,10.1007/s100520050537. [pp4.5]

[27] S. Heinemeyer, W. Hollik, G. Weiglein, The mass of the lightest MSSM Higgs boson: A compact analytical expression at the two loop level, Phys. Lett. B455 (1999) 179-191. arXiv:hep-ph/ 9903404, doi:10.1016/S0370-2693(99)00417-7.

[28] J. R. Espinosa, R.-J. Zhang, MSSM lightest CP even Higgs boson mass to $O\left(\alpha_{s} \alpha_{t}\right)$ : The effective potential approach, JHEP 03 (2000) 026. arXiv:hep-ph/9912236, doi:10.1088/1126-6708/ 2000/03/026

[29] M. Carena, H. E. Haber, S. Heinemeyer, W. Hollik, C. E. M. Wagner, G. Weiglein, Reconciling the two loop diagrammatic and effective field theory computations of the mass of the lightest CP - even Higgs boson in the MSSM, Nucl. Phys. B580 (2000) 29-57. arXiv:hep-ph/0001002, doi:10.1016/S0550-3213(00)00212-1.

[30] J. R. Espinosa, R.-J. Zhang, Complete two loop dominant corrections to the mass of the lightest CP even Higgs boson in the minimal supersymmetric standard model, Nucl. Phys. B586 (2000) 3-38. arXiv:hep-ph/0003246, doi:10.1016/S0550-3213(00)00421-1

[31] J. R. Espinosa, I. Navarro, Radiative corrections to the Higgs boson mass for a hierarchical stop spectrum, Nucl. Phys. B615 (2001) 82-116. arXiv:hep-ph/0104047, doi:10.1016/ S0550-3213(01) 00429-1.

[32] G. Degrassi, P. Slavich, F. Zwirner, On the neutral Higgs boson masses in the MSSM for arbitrary stop mixing, Nucl. Phys. B611 (2001) 403-422. arXiv:hep-ph/0105096, doi:10.1016/ S0550-3213(01) 00343-1. [p 5$]$

[33] S. P. Martin, Two loop effective potential for a general renormalizable theory and softly broken supersymmetry, Phys. Rev. D65 (2002) 116003. arXiv:hep-ph/0111209, doi:10.1103/ PhysRevD.65.116003

[34] A. Brignole, G. Degrassi, P. Slavich, F. Zwirner, On the $O\left(\alpha_{t}^{2}\right)$ two loop corrections to the neutral Higgs boson masses in the MSSM, Nucl. Phys. B631 (2002) 195-218. arXiv:hep-ph/0112177, doi:10.1016/S0550-3213(02)00184-0. [p 5]

[35] A. Brignole, G. Degrassi, P. Slavich, F. Zwirner, On the two loop sbottom corrections to the 
neutral Higgs boson masses in the MSSM, Nucl. Phys. B643 (2002) 79-92. arXiv:hep-ph/ 0206101, doi:10.1016/S0550-3213(02)00748-4. [pp 5,11

[36] S. P. Martin, Two loop effective potential for the minimal supersymmetric standard model, Phys. Rev. D66 (2002) 096001. arXiv:hep-ph/0206136, doi:10.1103/PhysRevD.66.096001.

[37] S. P. Martin, Complete two loop effective potential approximation to the lightest Higgs scalar boson mass in supersymmetry, Phys. Rev. D67 (2003) 095012. arXiv:hep-ph/0211366, doi: 10.1103/PhysRevD.67.095012

[38] G. Degrassi, S. Heinemeyer, W. Hollik, P. Slavich, G. Weiglein, Towards high precision predictions for the MSSM Higgs sector, Eur. Phys. J. C28 (2003) 133-143. arXiv:hep-ph/0212020, doi:10.1140/epjc/s2003-01152-2. [pp 4, 5, 6]

[39] A. Dedes, P. Slavich, Two loop corrections to radiative electroweak symmetry breaking in the MSSM, Nucl. Phys. B657 (2003) 333-354. arXiv:hep-ph/0212132, doi:10.1016/ S0550-3213(03) 00173-1.

[40] A. Dedes, G. Degrassi, P. Slavich, On the two loop Yukawa corrections to the MSSM Higgs boson masses at large $\tan \beta$, Nucl. Phys. B672 (2003) 144-162. arXiv:hep-ph/0305127, doi: 10.1016/j.nuclphysb.2003.08.033. [p 5

[41] S. P. Martin, Evaluation of two loop selfenergy basis integrals using differential equations, Phys. Rev. D68 (2003) 075002. arXiv:hep-ph/0307101, doi:10.1103/PhysRevD.68.075002

[42] S. P. Martin, Two loop scalar self energies in a general renormalizable theory at leading order in gauge couplings, Phys. Rev. D70 (2004) 016005. arXiv:hep-ph/0312092, doi:10.1103/ PhysRevD.70.016005.

[43] S. P. Martin, Strong and Yukawa two-loop contributions to Higgs scalar boson self-energies and pole masses in supersymmetry, Phys. Rev. D71 (2005) 016012. arXiv:hep-ph/0405022, doi:10.1103/PhysRevD.71.016012. [p 4

[44] B. C. Allanach, A. Djouadi, J. L. Kneur, W. Porod, P. Slavich, Precise determination of the neutral Higgs boson masses in the MSSM, JHEP 09 (2004) 044. arXiv:hep-ph/0406166, doi: 10.1088/1126-6708/2004/09/044.

[45] S. Heinemeyer, W. Hollik, H. Rzehak, G. Weiglein, High-precision predictions for the MSSM Higgs sector at $O\left(\alpha_{b} \alpha_{s}\right)$, Eur. Phys. J. C39 (2005) 465-481. arXiv:hep-ph/0411114, doi: 10.1140/epjc/s2005-02112-6. [pp5. 11]

[46] S. Heinemeyer, W. Hollik, G. Weiglein, Electroweak precision observables in the minimal supersymmetric standard model, Phys. Rept. 425 (2006) 265-368. arXiv:hep-ph/0412214, doi:10.1016/j.physrep.2005.12.002. [p 8]

[47] S. P. Martin, D. G. Robertson, TSIL: A Program for the calculation of two-loop self-energy integrals, Comput. Phys. Commun. 174 (2006) 133-151. arXiv:hep-ph/0501132, doi:10.1016/ j.cpc.2005.08.005

[48] S. P. Martin, Two-loop scalar self-energies and pole masses in a general renormalizable theory with massless gauge bosons, Phys. Rev. D71 (2005) 116004. arXiv:hep-ph/0502168, doi: 10.1103/PhysRevD.71.116004.

[49] S. P. Martin, Three-loop corrections to the lightest Higgs scalar boson mass in supersymmetry, Phys. Rev. D75 (2007) 055005. arXiv:hep-ph/0701051, doi:10.1103/PhysRevD.75.055005. $[\mathrm{p} 4$

[50] R. V. Harlander, P. Kant, L. Mihaila, M. Steinhauser, Higgs boson mass in supersymmetry to three loops, Phys. Rev. Lett. 100 (2008) 191602, [Phys. Rev. Lett.101,039901(2008)]. arXiv: 0803.0672, doi:10.1103/PhysRevLett.101.039901,10.1103/PhysRevLett.100.191602.

[51] P. Kant, R. V. Harlander, L. Mihaila, M. Steinhauser, Light MSSM Higgs boson mass to threeloop accuracy, JHEP 08 (2010) 104. arXiv:1005.5709, doi:10.1007/JHEP08(2010) 104

[52] T. Hahn, S. Heinemeyer, W. Hollik, H. Rzehak, G. Weiglein, High-precision predictions for the light CP-even Higgs boson mass of the Minimal Supersymmetric Standard Model, Phys. Rev. Lett. 112 (14) (2014) 141801. arXiv:1312.4937, doi:10.1103/PhysRevLett.112.141801. $[\mathrm{pp} 4.5,6$ 
[53] P. Draper, G. Lee, C. E. M. Wagner, Precise estimates of the Higgs mass in heavy supersymmetry, Phys. Rev. D89 (5) (2014) 055023. arXiv:1312.5743, doi:10.1103/PhysRevD.89.055023. [p 5

[54] S. Borowka, T. Hahn, S. Heinemeyer, G. Heinrich, W. Hollik, Momentum-dependent two-loop QCD corrections to the neutral Higgs-boson masses in the MSSM, Eur. Phys. J. C74 (8) (2014) 2994. arXiv:1404.7074, doi:10.1140/epjc/s10052-014-2994-0. [pp5, 12

[55] E. Bagnaschi, G. F. Giudice, P. Slavich, A. Strumia, Higgs mass and unnatural supersymmetry, JHEP 09 (2014) 092. arXiv:1407.4081, doi:10.1007/JHEP09(2014)092. [pp 5, 13

[56] G. Degrassi, S. Di Vita, P. Slavich, Two-loop QCD corrections to the MSSM Higgs masses beyond the effective-potential approximation, Eur. Phys. J. C75 (2) (2015) 61. arXiv:1410.3432, doi:10.1140/epjc/s10052-015-3280-5.

[57] J. P. Vega, G. Villadoro, SusyHD: Higgs mass determination in supersymmetry, JHEP 07 (2015) 159. arXiv: 1504.05200, doi:10.1007/JHEP07(2015) 159. [p]

[58] S. Borowka, T. Hahn, S. Heinemeyer, G. Heinrich, W. Hollik, Renormalization scheme dependence of the two-loop QCD corrections to the neutral Higgs-boson masses in the MSSM, Eur. Phys. J. C75 (9) (2015) 424. arXiv:1505.03133, doi:10.1140/epjc/s10052-015-3648-6. $[\mathrm{pp} 5,12$

[59] G. Lee, C. E. M. Wagner, Higgs bosons in heavy supersymmetry with an intermediate $\mathrm{m}_{A}$, Phys. Rev. D92 (7) (2015) 075032. arXiv:1508.00576, doi:10.1103/PhysRevD.92.075032. [pp 5, 14

[60] H. Bahl, W. Hollik, Precise prediction for the light MSSM Higgs boson mass combining effective field theory and fixed-order calculations, Eur. Phys. J. C76 (9) (2016) 499. arXiv:1608.01880 doi:10.1140/epjc/s10052-016-4354-8, [pp4,5, 14

[61] P. Athron, J.-H. Park, T. Steudtner, D. Stöckinger, A. Voigt, Precise Higgs mass calculations in (non-)minimal supersymmetry at both high and low scales, JHEP 01 (2017) 079. arXiv: 1609.00371, doi:10.1007/JHEP01(2017)079

[62] E. Bagnaschi, J. Pardo Vega, P. Slavich, Improved determination of the Higgs mass in the MSSM with heavy superpartners, Eur. Phys. J. C77 (5) (2017) 334. arXiv:1703.08166, doi: 10.1140/epjc/s10052-017-4885-7. [pp5 13

[63] H. Bahl, S. Heinemeyer, W. Hollik, G. Weiglein, Reconciling EFT and hybrid calculations of the light MSSM Higgs-boson mass, Eur. Phys. J. C78 (1) (2018) 57. arXiv:1706.00346, doi: 10.1140/epjc/s10052-018-5544-3. [pp4,5, 10,17

[64] R. V. Harlander, J. Klappert, A. Voigt, Higgs mass prediction in the MSSM at three-loop level in a pure $\overline{\mathrm{DR}}$ context, Eur. Phys. J. C77 (12) (2017) 814. arXiv:1708.05720, doi:10.1140/ epjc/s10052-017-5368-6.

[65] P. Athron, M. Bach, D. Harries, T. Kwasnitza, J.-H. Park, D. Stöckinger, A. Voigt, J. Ziebell, FlexibleSUSY 2.0: Extensions to investigate the phenomenology of SUSY and non-SUSY models, Comput. Phys. Commun. 230 (2018) 145-217. arXiv:1710.03760, doi:10.1016/j.cpc.2018. 04.016

[66] B. C. Allanach, A. Voigt, Uncertainties in the Lightest $C P$ Even Higgs Boson Mass Prediction in the Minimal Supersymmetric Standard Model: Fixed Order Versus Effective Field Theory Prediction, Eur. Phys. J. C78 (7) (2018) 573. arXiv:1804.09410, doi:10.1140/epjc/ s10052-018-6046-z.

[67] H. Bahl, W. Hollik, Precise prediction of the MSSM Higgs boson masses for low $\mathrm{M}_{A}$, JHEP 07 (2018) 182. arXiv: 1805.00867, doi:10.1007/JHEP07 (2018) 182. [pp 5,6, 17,18

[68] R. V. Harlander, J. Klappert, A. D. Ochoa Franco, A. Voigt, The light CP-even MSSM Higgs mass resummed to fourth logarithmic order. arXiv:1807.03509. [pp 4 5

[69] A. Pilaftsis, CP odd tadpole renormalization of Higgs scalar - pseudoscalar mixing, Phys. Rev. D58 (1998) 096010. arXiv:hep-ph/9803297, doi:10.1103/PhysRevD.58.096010. [p 4]

[70] D. A. Demir, Effects of the supersymmetric phases on the neutral Higgs sector, Phys. Rev. D60 (1999) 055006. arXiv:hep-ph/9901389, doi:10.1103/PhysRevD.60.055006.

[71] A. Pilaftsis, C. E. M. Wagner, Higgs bosons in the minimal supersymmetric standard model with explicit CP violation, Nucl. Phys. B553 (1999) 3-42. arXiv:hep-ph/9902371, doi:10.1016/ 
S0550-3213(99)00261-8

[72] S. Y. Choi, M. Drees, J. S. Lee, Loop corrections to the neutral Higgs boson sector of the MSSM with explicit CP violation, Phys. Lett. B481 (2000) 57-66. arXiv:hep-ph/0002287, doi:10.1016/S0370-2693(00)00421-4.

[73] M. Carena, J. R. Ellis, A. Pilaftsis, C. E. M. Wagner, Renormalization group improved effective potential for the MSSM Higgs sector with explicit CP violation, Nucl. Phys. B586 (2000) 92-140. arXiv:hep-ph/0003180, doi:10.1016/S0550-3213(00)00358-8,

[74] T. Ibrahim, P. Nath, Corrections to the Higgs boson masses and mixings from chargino, W and charged Higgs exchange loops and large CP phases, Phys. Rev. D63 (2001) 035009. arXiv: hep-ph/0008237, doi:10.1103/PhysRevD.63.035009.

[75] S. Heinemeyer, The Higgs boson sector of the complex MSSM in the Feynman diagrammatic approach, Eur. Phys. J. C22 (2001) 521-534. arXiv:hep-ph/0108059, doi:10.1007/ s100520100819.

[76] T. Ibrahim, P. Nath, Neutralino exchange corrections to the Higgs boson mixings with explicit CP violation, Phys. Rev. D66 (2002) 015005. arXiv:hep-ph/0204092, doi:10.1103/PhysRevD. 66.015005 .

[77] M. Frank, T. Hahn, S. Heinemeyer, W. Hollik, H. Rzehak, G. Weiglein, The Higgs boson masses and mixings of the complex MSSM in the Feynman-diagrammatic approach, JHEP 02 (2007) 047. arXiv:hep-ph/0611326, doi:10.1088/1126-6708/2007/02/047. [pp 4, 5, 6, 9

[78] S. Heinemeyer, W. Hollik, H. Rzehak, G. Weiglein, The Higgs sector of the complex MSSM at two-loop order: QCD contributions, Phys. Lett. B652 (2007) 300-309. arXiv:0705.0746, doi:10.1016/j.physletb.2007.07.030. [pp5,9]

[79] W. Hollik, S. Paßehr, Two-loop top-Yukawa-coupling corrections to the Higgs boson masses in the complex MSSM, Phys. Lett. B733 (2014) 144-150. arXiv:1401.8275, doi:10.1016/j. physletb.2014.04.026.

[80] W. Hollik, S. Paßehr, Higgs boson masses and mixings in the complex MSSM with twoloop top-Yukawa-coupling corrections, JHEP 10 (2014) 171. arXiv:1409.1687, doi:10.1007/ JHEP10 (2014) 171. [pp5, 12]

[81] M. D. Goodsell, F. Staub, The Higgs mass in the CP violating MSSM, NMSSM, and beyond, Eur. Phys. J. C77 (1) (2017) 46. arXiv:1604.05335, doi:10.1140/epjc/s10052-016-4495-9.

[82] S. Paßehr, G. Weiglein, Two-loop top and bottom Yukawa corrections to the Higgs-boson masses in the complex MSSM, Eur. Phys. J. C78 (3) (2018) 222. arXiv:1705.07909, doi:10.1140/ epjc/s10052-018-5665-8. [p 16.

[83] S. Borowka, S. Paßehr, G. Weiglein, Complete two-loop QCD contributions to the lightest Higgsboson mass in the MSSM with complex parameters, Eur. Phys. J. C78 (7) (2018) 576. arXiv: 1802.09886, doi:10.1140/epjc/s10052-018-6055-y. [pp 4, 5,16

[84] S. Heinemeyer, W. Hollik, G. Weiglein, FeynHiggs: A Program for the calculation of the masses of the neutral CP even Higgs bosons in the MSSM, Comput. Phys. Commun. 124 (2000) 76-89. arXiv:hep-ph/9812320, doi:10.1016/S0010-4655(99)00364-1. [pp45

[85] T. Hahn, S. Heinemeyer, W. Hollik, H. Rzehak, G. Weiglein, FeynHiggs: A program for the calculation of MSSM Higgs-boson observables - Version 2.6.5, Comput. Phys. Commun. 180 (2009) 1426-1427. doi:10.1016/j.cpc.2009.02.014. [pp 4 5]

[86] D. de Florian, et al., Handbook of LHC Higgs Cross Sections: 4. Deciphering the Nature of the Higgs Sector. arXiv:1610.07922, doi:10.23731/CYRM-2017-002, [pp 4, 8

[87] S. Heinemeyer, et al., Handbook of LHC Higgs Cross Sections: 3. Higgs Properties. arXiv: 1307.1347, doi:10.5170/CERN-2013-004. [pp 4, 8]

[88] H. Bahl, DESY-18-188, MPP-2018-240 (in preparation). [p 4

[89] W. Hollik, S. Paßehr, Two-loop top-Yukawa-coupling corrections to the charged Higgs-boson mass in the MSSM, Eur. Phys. J. C75 (7) (2015) 336. arXiv:1502.02394, doi:10.1140/epjc/ s10052-015-3558-7. [p 5]

[90] T. Hahn, S. Paßehr, Implementation of the $\mathcal{O}\left(\alpha_{t}^{2}\right)$ MSSM Higgs-mass corrections in FeynHiggs, 
Comput. Phys. Commun. 214 (2017) 91-97. arXiv:1508.00562, doi:10.1016/j.cpc.2017. 01.026 . [pp 5 . 15

[91] M. Arana-Catania, S. Heinemeyer, M. J. Herrero, S. Penaranda, Higgs Boson masses and BPhysics Constraints in Non-Minimal Flavor Violating SUSY scenarios, JHEP 05 (2012) 015. arXiv:1109.6232, doi:10.1007/JHEP05(2012)015, [p 5]

[92] S. Heinemeyer, W. Hollik, F. Merz, S. Penaranda, Electroweak precision observables in the MSSM with nonminimal flavor violation, Eur. Phys. J. C37 (2004) 481-493. arXiv:hep-ph/ 0403228, doi:10.1140/epjc/s2004-02006-1.

[93] M. E. Gómez, T. Hahn, S. Heinemeyer, M. Rehman, Higgs masses and Electroweak Precision Observables in the Lepton-Flavor-Violating MSSM, Phys. Rev. D90 (7) (2014) 074016. arXiv: 1408.0663, doi:10.1103/PhysRevD.90.074016. [p 5]

[94] G. F. Giudice, A. Strumia, Probing high-scale and split supersymmetry with Higgs mass measurements, Nucl. Phys. B858 (2012) 63-83. arXiv:1108.6077, doi:10.1016/j.nuclphysb. 2012.01.001. [p 5]

[95] S. Heinemeyer, W. Hollik, J. Rosiek, G. Weiglein, Neutral MSSM Higgs boson production at e+ e- colliders in the Feynman diagrammatic approach, Eur. Phys. J. C19 (2001) 535-546. arXiv:hep-ph/0102081, doi:10.1007/s100520100631. [p6]

[96] E. Fuchs, G. Weiglein, Breit-Wigner approximation for propagators of mixed unstable states, JHEP 09 (2017) 079. arXiv:1610.06193, doi:10.1007/JHEP09(2017)079.

[97] E. Fuchs, G. Weiglein, Impact of CP-violating interference effects on MSSM Higgs searches, Eur. Phys. J. C78 (2) (2018) 87. arXiv:1705.05757, doi:10.1140/epjc/s10052-018-5543-4. [p 6]

[98] F. Domingo, P. Drechsel, S. Paßehr, On-Shell neutral Higgs bosons in the NMSSM with complex parameters, Eur. Phys. J. C77 (8) (2017) 562. arXiv:1706.00437, doi:10.1140/epjc/ s10052-017-5104-2. [pp 6 16

[99] E. Fuchs, Interference effects in new physics processes at the LHC, Ph.D. thesis, U. Hamburg, Dept. Phys., Hamburg (2015). doi:10.3204/DESY-THESIS-2015-037. URL http://bib-pubdb1.desy.de/record/224288 [p 6]

[100] S. Heinemeyer, W. Hollik, G. Weiglein, Decay widths of the neutral CP even MSSM Higgs bosons in the Feynman diagrammatic approach, Eur. Phys. J. C16 (2000) 139-153. arXiv: hep-ph/0003022, doi:10.1007/s100520050010. [p 6]

[101] F. Domingo, S. Heinemeyer, S. Paßehr, G. Weiglein, Decays of the neutral Higgs bosons into SM fermions and gauge bosons in the $\mathcal{C} \mathcal{P}$-violating NMSSMarXiv:1807.06322. [pp]7] 16$]$

[102] M. Spira, A. Djouadi, D. Graudenz, P. M. Zerwas, Higgs boson production at the LHC, Nucl. Phys. B453 (1995) 17-82. arXiv:hep-ph/9504378, doi:10.1016/0550-3213(95) 00379-7, [p]

[103] U. Aglietti, R. Bonciani, G. Degrassi, A. Vicini, Analytic Results for Virtual QCD Corrections to Higgs Production and Decay, JHEP 01 (2007) 021. arXiv:hep-ph/0611266, doi:10.1088/ $1126-6708 / 2007 / 01 / 021$.

[104] R. Benbrik, M. Gomez Bock, S. Heinemeyer, O. Stal, G. Weiglein, L. Zeune, Confronting the MSSM and the NMSSM with the Discovery of a Signal in the two Photon Channel at the LHC, Eur. Phys. J. C72 (2012) 2171. arXiv:1207.1096, doi:10.1140/epjc/s10052-012-2171-2. $[\mathrm{p} 7]$

[105] A. Bredenstein, A. Denner, S. Dittmaier, M. M. Weber, Radiative corrections to the semileptonic and hadronic Higgs-boson decays $H \rightarrow W W / Z Z \rightarrow 4$ fermions, JHEP 02 (2007) 080. arXiv: hep-ph/0611234, doi:10.1088/1126-6708/2007/02/080, [p]

[106] A. Bredenstein, A. Denner, S. Dittmaier, M. M. Weber, Precise predictions for the Higgs-boson decay $H \rightarrow W W / Z Z \rightarrow 4$ leptons, Phys. Rev. D74 (2006) 013004. arXiv:hep-ph/0604011, doi:10.1103/PhysRevD.74.013004. [p]

[107] K. E. Williams, H. Rzehak, G. Weiglein, Higher order corrections to Higgs boson decays in the MSSM with complex parameters, Eur. Phys. J. C71 (2011) 1669. arXiv:1103.1335, doi: 10.1140/epjc/s10052-011-1669-3. [pp7] 11 
[108] A. Djouadi, J. Kalinowski, P. M. Zerwas, Two and three-body decay modes of SUSY Higgs particles, Z. Phys. C70 (1996) 435-448. arXiv:hep-ph/9511342, doi:10.1007/s002880050121. $[\mathrm{p} 7]$

[109] K. E. Williams, G. Weiglein, Precise predictions for $h_{a} \rightarrow h_{b} h_{c}$ decays in the complex MSSM, Phys. Lett. B660 (2008) 217-227. arXiv:0710.5320, doi:10.1016/j.physletb.2007.12.049. [pp 7, 11$]$

[110] R. V. Harlander, W. B. Kilgore, Higgs boson production in bottom quark fusion at next-tonext-to leading order, Phys. Rev. D68 (2003) 013001. arXiv:hep-ph/0304035, doi:10.1103/ PhysRevD.68.013001. [p 8

[111] E. L. Berger, T. Han, J. Jiang, T. Plehn, Associated production of a top quark and a charged Higgs boson, Phys. Rev. D71 (2005) 115012. arXiv:hep-ph/0312286, doi:10.1103/PhysRevD. 71.115012. $[\mathrm{p} 8$

[112] S. Dittmaier, M. Krämer, M. Spira, M. Walser, Charged-Higgs-boson production at the LHC: NLO supersymmetric QCD corrections, Phys. Rev. D83 (2011) 055005. arXiv:0906.2648, doi: 10.1103/PhysRevD.83.055005.

[113] M. Flechl, R. Klees, M. Krämer, M. Spira, M. Ubiali, Improved cross-section predictions for heavy charged Higgs boson production at the LHC, Phys. Rev. D91 (7) (2015) 075015. arXiv: 1409.5615, doi:10.1103/PhysRevD.91.075015.

[114] C. Degrande, M. Ubiali, M. Wiesemann, M. Zaro, Heavy charged Higgs boson production at the LHC, JHEP 10 (2015) 145. arXiv:1507.02549, doi:10.1007/JHEP10(2015) 145. [p 8

[115] M. Carena, D. Garcia, U. Nierste, C. E. M. Wagner, Effective Lagrangian for the $\bar{t} b H^{+}$interaction in the MSSM and charged Higgs phenomenology, Nucl. Phys. B577 (2000) 88-120. arXiv: hep-ph/9912516, doi:10.1016/S0550-3213(00)00146-2, [p 8]

[116] J. G. Körner, M. C. Mauser, $O\left(\alpha_{s}\right)$ radiative corrections to polarized top decay into a charged Higgs, $t^{\uparrow} \rightarrow H^{+}+b$, Eur. Phys. J. C54 (2008) 175-185. arXiv:hep-ph/0211098, doi:10.1140/ epjc/s10052-007-0510-5. [p 8

[117] S. Heinemeyer, W. Hollik, D. Stöckinger, A. M. Weber, G. Weiglein, Precise prediction for M(W) in the MSSM, JHEP 08 (2006) 052. arXiv:hep-ph/0604147, doi:10.1088/1126-6708/2006/ 08/052. [p 8

[118] S. Heinemeyer, W. Hollik, G. Weiglein, L. Zeune, Implications of LHC search results on the W boson mass prediction in the MSSM, JHEP 12 (2013) 084. arXiv:1311.1663, doi:10.1007/ JHEP12 (2013) 084. [p 8

[119] O. Stål, G. Weiglein, L. Zeune, Improved prediction for the mass of the W boson in the NMSSM, JHEP 09 (2015) 158. arXiv:1506.07465, doi:10.1007/JHEP09(2015)158. [p8]

[120] S. Heinemeyer, W. Hollik, A. M. Weber, G. Weiglein, $Z$ Pole Observables in the MSSM, JHEP 04 (2008) 039. arXiv:0710.2972, doi:10.1088/1126-6708/2008/04/039. [p 8

[121] G. Degrassi, G. F. Giudice, QED logarithms in the electroweak corrections to the muon anomalous magnetic moment, Phys. Rev. D58 (1998) 053007. arXiv:hep-ph/9803384, doi: 10.1103/PhysRevD.58.053007. [p 8

[122] S. Heinemeyer, D. Stöckinger, G. Weiglein, Two loop SUSY corrections to the anomalous magnetic moment of the muon, Nucl. Phys. B690 (2004) 62-80. arXiv:hep-ph/0312264. doi:10.1016/j.nuclphysb.2004.04.017. [p 8

[123] T. Ibrahim, P. Nath, The Neutron and the electron electric dipole moment in $\mathrm{N}=1$ supergravity unification, Phys. Rev. D57 (1998) 478-488, [Erratum: Phys. Rev.D60,119901(1999)]. arXiv:hep-ph/9708456, doi:10.1103/PhysRevD.58.019901,10.1103/PhysRevD.60.079903, 10.1103/PhysRevD.60.119901, 10.1103/PhysRevD.57.478. $\mid \mathrm{p}[8$

[124] D. A. Demir, O. Lebedev, K. A. Olive, M. Pospelov, A. Ritz, Electric dipole moments in the MSSM at large tan beta, Nucl. Phys. B680 (2004) 339-374. arXiv:hep-ph/0311314, doi: $10.1016 / \mathrm{j}$. nuclphysb.2003.12.026

[125] D. Chang, W. Keung, A. Pilaftsis, New two loop contribution to electric dipole moment 
in supersymmetric theories, Phys. Rev. Lett. 82 (1999) 900-903, [Erratum: Phys. Rev. Lett.83,3972(1999)]. arXiv:hep-ph/9811202, doi:10.1103/PhysRevLett.83.3972,10.1103/ PhysRevLett.82.900

[126] K. A. Olive, M. Pospelov, A. Ritz, Y. Santoso, CP-odd phase correlations and electric dipole moments, Phys. Rev. D72 (2005) 075001. arXiv:hep-ph/0506106, doi:10.1103/PhysRevD. 72.075001. [p 8

[127] T. Hahn, W. Hollik, J. I. Illana, S. Penaranda, Interplay between $\mathrm{H} \rightarrow \mathrm{b}$ anti-s and $\mathrm{b} \rightarrow \mathrm{s}$ gamma in the MSSM with non-minimal flavor violationarXiv:hep-ph/0512315. [p] 8

[128] A. J. Buras, S. Jäger, J. Urban, Master formulae for Delta $F=2$ NLO QCD factors in the standard model and beyond, Nucl. Phys. B605 (2001) 600-624. arXiv:hep-ph/0102316, doi: 10.1016/S0550-3213(01)00207-3. [p 8]

[129] C. Bobeth, A. J. Buras, F. Kruger, J. Urban, QCD corrections to $\bar{B} \rightarrow X_{d, s} \nu \bar{\nu}, \bar{B}_{d, s} \rightarrow \ell^{+} \ell^{-}$, $K \rightarrow \pi \nu \bar{\nu}$ and $K_{L} \rightarrow \mu^{+} \mu^{-}$in the MSSM, Nucl. Phys. B630 (2002) 87-131. arXiv:hep-ph/ 0112305, doi:10.1016/S0550-3213(02)00141-4. [p 8]

[130] M. Carena, J. Ellis, J. S. Lee, A. Pilaftsis, C. E. M. Wagner, CP Violation in Heavy MSSM Higgs Scenarios, JHEP 02 (2016) 123. arXiv:1512.00437, doi:10.1007/JHEP02(2016) 123. [p 14]

[131] T. Hahn, M. Perez-Victoria, Automated one loop calculations in four-dimensions and Ddimensions, Comput. Phys. Commun. 118 (1999) 153-165. arXiv:hep-ph/9807565, doi: 10.1016/S0010-4655(98)00173-8. [pp 14, 16

[132] T. Hahn, SUSY Les Houches Accord 2 I/O made easy, Comput. Phys. Commun. 180 (2009) 1681-1693. arXiv:hep-ph/0605049, doi:10.1016/j.cpc.2009.03.012. [p 14]

[133] T. Fritzsche, T. Hahn, S. Heinemeyer, F. von der Pahlen, H. Rzehak, C. Schappacher, The Implementation of the Renormalized Complex MSSM in FeynArts and FormCalc, Comput. Phys. Commun. 185 (2014) 1529-1545. arXiv:1309.1692, doi:10.1016/j.cpc.2014.02.005. [p 16]

[134] T. Hahn, Generating Feynman diagrams and amplitudes with FeynArts 3, Comput. Phys. Commun. 140 (2001) 418-431. arXiv:hep-ph/0012260, doi:10.1016/S0010-4655(01)00290-9. $[\mathrm{p} 16]$

[135] P. Drechsel, L. Galeta, S. Heinemeyer, G. Weiglein, Precise Predictions for the Higgs-Boson Masses in the NMSSM, Eur. Phys. J. C77 (1) (2017) 42. arXiv:1601.08100, doi:10.1140/ epjc/s10052-017-4595-1. [p 16

[136] H. Bahl, E. Fuchs, T. Hahn, S. Heinemeyer, S. Liebler, S. Patel, P. Slavich, T. Stefaniak, C. E. M. Wagner, G. Weiglein, MSSM Higgs Boson Searches at the LHC: Benchmark Scenarios for Run 2 and Beyond. arXiv:1808.07542. [p 17

[137] E. Bagnaschi, et al., Supersymmetric Models in Light of Improved Higgs Mass Calculations. arXiv: 1810.10905. [pp 17, 18 